\title{
Meiotic chromosome configurations in triploid progeny from reciprocal crosses between wild-type diploid and natural tetraploid loach Misgurnus anguillicaudatus in China
}

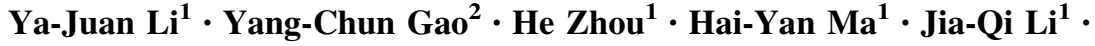 \\ Katsutoshi Arai $^{3}$
}

Received: 3 February 2015/Accepted: 21 June 2015/Published online: 1 July 2015

(c) Springer International Publishing Switzerland 2015

\begin{abstract}
Here, we showed meiotic chromosome configurations prepared from oocyte germinal vesicles and spermatocytes of triploid loaches produced from reciprocal crosses between wild-type diploids $(2 \mathrm{n}=50)$ obtained from Dalian, Liaoning Province, China and natural tetraploids $(4 \mathrm{n}=100)$ from Chibi, Hubei Province, China. Major meiotic cells in triploids comprised 25 bivalents and 25 univalents, but cells with one to five trivalents were also observed. When three homologous chromosomes bearing nucleolar organizing regions (NOR) were identified with the detection of signals or positive sites by silver staining, chromomycin $\mathrm{A}_{3}$ staining and fluorescence in situ hybridization with a $5.8 \mathrm{~S}+28 \mathrm{~S}$ rDNA probe, two third of selected triploid cells gave a configuration including one bivalent with two NORs (association of two homologous chromosomes) and one univalent with one NOR. However, other triploid cells showed three univalent each of which had one NOR, suggesting a failure of synapsis between homologous chromosomes. These results suggested that
\end{abstract}

Electronic supplementary material The online version of this article (doi:10.1007/s10709-015-9853-2) contains supplementary material, which is available to authorized users.

Katsutoshi Arai

araikt@fish.hokudai.ac.jp

1 Key Laboratory of Mariculture and Stock Enhancement in North China's Sea, Ministry of Agriculture, Dalian Ocean University, Dalian 116023, China

2 Research Center for Eco-Environmental Science, Chinese Academy of Science, 18 Shuangqing Road, Haidian District, Beijing 100085, China

3 Faculty and Graduate School of Fisheries Sciences, Hokkaido University, 3-1-1, Minato, Hakodate, Hokkaido 041-8611, Japan triploid female and male should produce aneuploid gametes with the theoretical mode at $1.5 \mathrm{n}$ ( 37 or 38 chromosomes).

Keywords Bivalent · Gamete - Hybrid · Polyploid · Trivalent · Univalent

\section{Introduction}

When diploid females exhibit fully mature ovary, most oocytes cannot enter into vitellogenesis in the ovary of artificially induced triploid fishes (Benfey 1999, 2011; Piferrer et al. 2009). Ovarian development is largely retarded in induced triploid females in several teleosts (Benfey 1999, 2011; Piferrer et al. 2009). On the contrary, induced triploid males often generate a small quantity of aneuploid spermatozoa because testis develops to some extent and secondary sex characters arise (Benfey 1999, 2011; Piferrer et al. 2009). These reproductive characteristics are also the cases in artificially induced triploid loach or Oriental weatherfish, Misgurnus anguillicaudatus (Suzuki et al. 1985; Zhang and Arai 1999; Arai and Fujimoto 2013). However, reproductive performance of spontaneous triploid fishes, which infrequently occur in nature, is different from that of artificially induced triploid fishes and their reproductive capacities are much more diversified (Arai and Fujimoto 2013).

In northern area of Hokkaido, Japan, natural triploid loaches infrequently arise from unreduced diploid eggs, which are spawned by the gynogenetically reproducing clonal diploid population, by accidental incorporation of a sperm nucleus into the egg (Morishima et al. 2002, 2008a; Itono et al. 2007). Such clone-origin triploid females mainly produce haploid eggs by the mechanism of meiotic 
hybridogenesis together with a small number of unreduced triploid eggs, but males are sterile (Morishima et al. 2008b; Arai and Fujimoto 2013).

Simultaneous production of unreduced triploid eggs and post-meiotic haploid eggs was also reported in triploid females which occurred from reciprocal crosses between wild-type diploids from Japanese population and natural tetraploids, which distributed in central part of China and presumably appeared in Japanese market samples, because Chinese loaches including such natural tetraploids are commercially imported to Japan as foods and live bait for fishing (Matsubara et al. 1995; Zhang and Arai 1996; Arai and Fujimoto 2013). It is generally accepted that unreduced triploid eggs are formed by the mechanism of premeiotic endomitosis (Arai and Mukaino 1997; Zhang et al. 1998), while haploid eggs by the meiotic hybridogenesis-like mechanism (Arai and Mukaino 1998). However, triploid males from the crosses between wild-type diploid and natural tetraploid could not generate fertile spermatozoa (Matsubara et al. 1995; Zhang and Arai 1996).

In Japanese wild populations, no tetraploid loach has been detected (Arai and Fujimoto 2013), but diploid-tetraploid complex exists in Chang Jiang River system in central China (Li et al. 2008, 2010). Li et al. (2012, 2013) verified gonochorisms and sexual reproduction both in Chinese diploid and tetraploid loaches: they reproduce by sexual fertilization of post-meiotic gametes (eggs and spermatozoa) and reciprocal hybridization between diploid and tetraploid produces viable triploid progeny. $\mathrm{Li}$ et al. (2012) also found sympatric occurrence of natural triploids and reported their reproductive performance. Spontaneous occurring triploid female produced fertile haploid eggs from which normal viable progeny appeared after fertilization with spermatozoa of diploid and tetraploid males (Li et al. 2012). On the other hand, a triploid male produced abnormal progeny with about $2.5 \mathrm{n}$ chromosomes, suggesting the generation of aneuploid spermatozoa by meiosis of natural triploid males (Li et al. 2012).

Configuration of meiotic chromosomes was an essential information to consider the cytogenetic mechanism involved in the generation of gametes in triploid organisms. In the present study, we observed meiotic configurations of chromosomes in both the oocyte germinal vesicles (GVs) and the spermatocytes of the triploid progeny from the reciprocal crosses between wild-type diploids and natural tetraploids in 2010 ( $\mathrm{Li}$ et al. 2012). In our study, the pairing behavior of homologous chromosomes (univalent, bivalents or other unusual multivalents) was carefully observed by the use of conventional Giemsa staining, silver staining of nucleolar organizing regions (Ag-NORs), chromomycin $\mathrm{A}_{3}\left(\mathrm{CMA}_{3}\right)$ differential staining and fluorescence in situ hybridization (FISH) using a $5.8 \mathrm{~S}+28 \mathrm{~S}$ rDNA probe $(\mathrm{Li}$ et al. 2010, 2011, 2013), in order to infer reproductive performance of triploids from the reciprocal crosses between wild-type diploid and natural tetraploid loaches.

\section{Materials and methods}

Natural tetraploid loaches $(4 n=100)$ were collected in Chibi, Hubei Province, China. Diploid loaches $(2 n=50)$ were collected in the fish market, Dalian, Liaoning Province, China. Ploidy status of each sample was determined by erythrocyte measurements and cellular DNA content flow cytometry ( $\mathrm{Li}$ et al. 2008), in advance. All the samples were kept in the aquarium of the Dalian Ocean University $\left(22 \pm 1{ }^{\circ} \mathrm{C}\right)$. In 2010 , crosses diploid female $\times$ tetraploid male $(2 n \times 4 n)$ and tetraploid female $\times$ diploid male $(4 n \times 2 n)$ were performed. Fertilized eggs from each cross and larvae were incubated at a temperature of $25 \pm 1^{\circ} \mathrm{C}$. Water was changed daily, air was also provided and died larvae were removed.

In early summer spawning season of 2012, cytogenetic analyses of oocyte germinal vesicles (GVs) were made on four females from $2 n \times 4 n$ crosses and five females from $4 n \times 2 n$ crosses following the procedure of Itono et al. (2006) and Li et al. (2011). Briefly, fragments including full grown immature oocytes with sizes similar to mature eggs were removed from the ovaries and incubated in goldfish saline containing 17 $\alpha-20 \beta$ dihidroxy-4-pregnene3-one (Sigma) at room temperature (Zhang et al. 1998). During germinal vesicle migration (GVM) but before germinal vesicle break down (GVBD), oocytes were fixed with Carnoy's fixative and GVs were isolated (Itono et al. 2006; Li et al. 2011). GVs were air-dried on a clean slide and then were observed after DAPI (4',6-diamidino-2phenylindole, Sigma) staining under a fluorescence microscope (Itono et al. 2006; Li et al. 2011).

In 2012, five males from $2 n \times 4 n$ and five from $4 n \times 2 n$ were injected twice with phytohemagglutinin-A as described in $\mathrm{Li}$ et al. (2011). The testes were removed and treated with a hypotonic solution ( $0.8 \%$ tri-sodium citrate). Then, the testes were fixed with Carnoy's fixative. A cell suspension was made from fixed testes of each male and then one droplet was pipetted on a slide glass. The slides were air-dried and stained with Giemsa (Merck) diluted with phosphate buffer.

Detection of homologous chromosomes bearing nuclear organizing regions (NORs) was made by silver nitrate staining (Ag-NOR) according to the method described in $\mathrm{Li}$ et al. (2013). The fluorochrome $\mathrm{CMA}_{3}$ (chromomycin $\mathrm{A}_{3}$, Wako)/DA (distamycin A, Sigma) staining was also performed to detect NORs according to $\mathrm{Li}$ et al. (2010, 2011, 2013). FISH with human $5.8 \mathrm{~S}+28 \mathrm{~S}$ rDNA as a probe was also conducted to detect NORs as described in $\mathrm{Li}$ et al. (2010, 2011, 2013). Signals were detected under a Leica DM2000, and the image was captured with DF 450C 
CCD camera and processed with the software package Adobe Photoshop (ver. 7.0).

\section{Results}

In four triploid females developed from the crosses $2 n \times 4 n, 15$ out of $51 \mathrm{GVs}$ gave a configuration including 25 bivalents (II) and 25 univalents (I) (Fig. 1a) and 16 out of $51 \mathrm{GVs}$ showed another configuration including 24II, 24I and 1 trivalent (III) (Figs. 1b, 2; Table S1). Typical trivalent chromosomes often exhibited long rod-like shape and triangular or ring-like configuration (Fig. 1b, c). Other triploid GVs exhibited 23I $+23 \mathrm{II}+2 \mathrm{III}, 22 \mathrm{I}+22 \mathrm{II}+3 \mathrm{III}$ and $21 \mathrm{I}+21 \mathrm{II}+4 \mathrm{III}$. Near triploid cells with extra chromosome numbers $(\mathrm{CN})$ of 77-82 and those with insufficient $\mathrm{CN}$ of 67-74 also appeared (Fig. 2). In five $4 n \times 2 n$ triploid females, 16 out of 25 triploid GVs showed clear mode with 25I and 25II (Fig. 2, Table S1). Other triploid cells had $24 \mathrm{I}+24 \mathrm{II}+1 \mathrm{III}$ and $23 \mathrm{I}+23 \mathrm{II}+2 \mathrm{III}$. A few GVs with $\mathrm{CN}$ of 64-71 and those with $\mathrm{CN}$ of 81 and 90 also occurred.
In five triploid males from $2 n \times 4 n$ cross, 48 out of 163 spermatocytes gave the mode with $25 \mathrm{I}+25 \mathrm{II}$ (Fig. 1d), but other triploid meiotic cells includes trivalents with long rodlike shape (Fig. 1e, f): 18 cells with $24 \mathrm{I}+24 \mathrm{II}+1 \mathrm{III}, 11$ cells with $23 \mathrm{I}+23 \mathrm{II}+2 \mathrm{III}, 4$ cells with $22 \mathrm{I}+22 \mathrm{II}+$ $3 \mathrm{III}$, one cell with $21 \mathrm{I}+21 \mathrm{II}+4 \mathrm{III}$, and one cell with $20 \mathrm{I}+20 \mathrm{II}+5 \mathrm{III}$ (Fig. 3; Table S2). In spermatocytes, relatively large numbers of cells with polyploid range with CN of 81-157 appeared together with cells with near triploid CN of 70-74 and 76-80 (Fig. 3; Table S2).

Similar configurations were observed in spermatocytes of five triploid males from $4 n \times 2 n$ cross (Fig. 3; Table S3). The modal triploid cells gave $25 \mathrm{I}+25 \mathrm{II}$ configuration (53 out of 264 cells). Other triploid spermatocytes were $24 \mathrm{I}+24 \mathrm{II}+1 \mathrm{III}$ (6 cells), $23 \mathrm{I}+23 \mathrm{II}+2 \mathrm{III}(6$ cells) and $23 \mathrm{I}+26 \mathrm{II}$ (2 cells). In testes of triploid $(4 n \times 2 n)$ males, cells with tetraploid range $\mathrm{CN}$ (84-116), pentaploid range $\mathrm{CN}$ (127-133) and hexaploid range $\mathrm{CN}$ (152) appeared together with near triploid CN (70-74, 76-82) with different meiotic configurations (Fig. 3; Table S3).
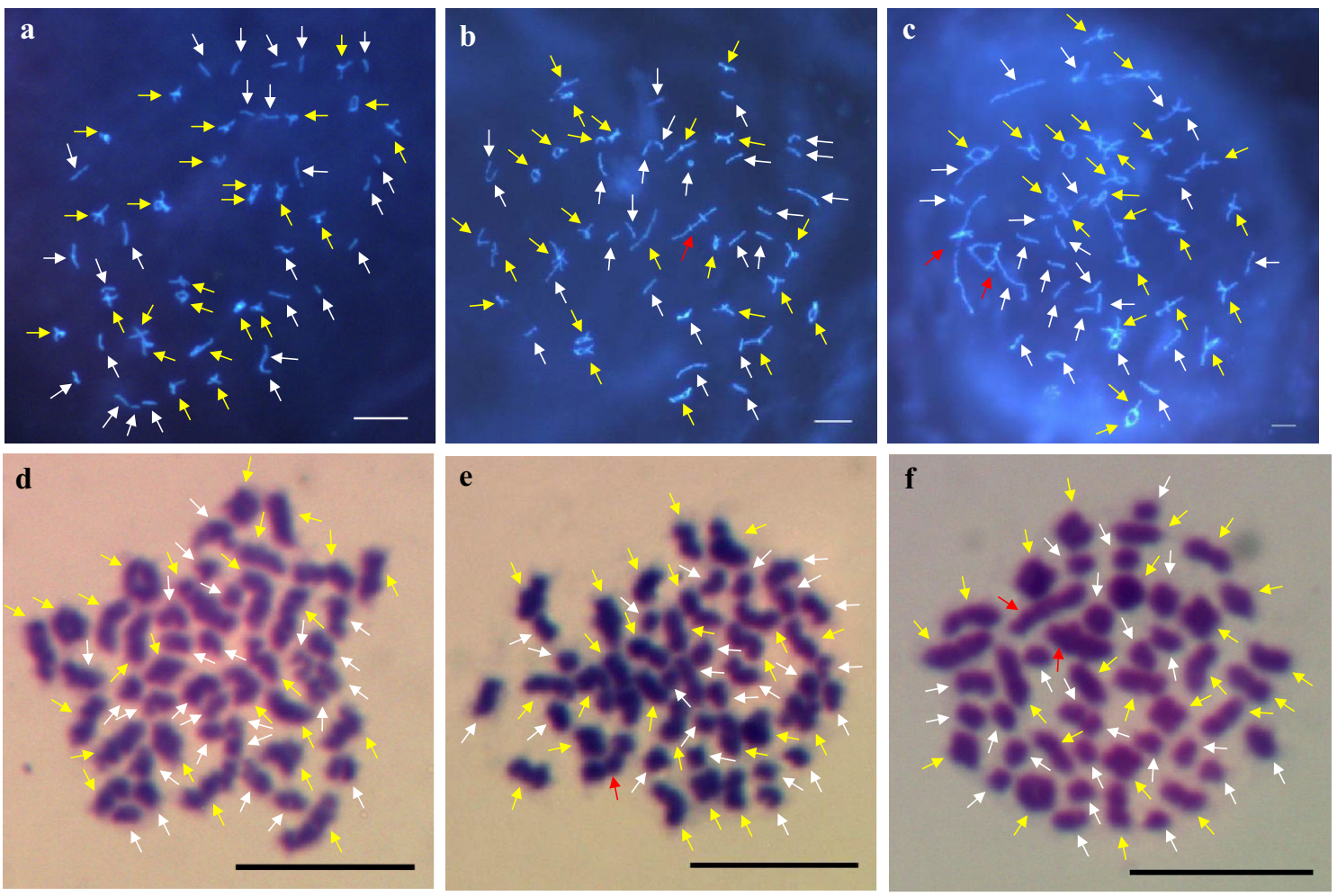

Fig. 1 Meiotic configurations in oocyte germinal vesicles of triploid females $(\mathbf{a}-\mathbf{c})$ and those in spermatocytes of triploid males $(\mathbf{d}-\mathbf{f})$ in loach Misgurnus anguillicaudatus. DAPI-stained germinal vesicles comprising 25 univalents (I) and 25 bivalents (II) (a), $24 \mathrm{I}+24 \mathrm{II}+$ one trivalent (III) (b), and $23 \mathrm{I}+23 \mathrm{II}+2$ III (c) prepared from triploid females. Conventional Giemsa-stained spermatocytes comprising 25 I and 25 II (d), 24I + 24II + 1 III (e), and $23 \mathrm{I}+23 \mathrm{II}+2$ III (f). White, yellow and red arrows denote univalents, bivalents and trivalents, respectively. Scales denote $10 \mu \mathrm{m}$ 
Fig. 2 Number of univalents (I), bivalents (II) and trivalents (III), and chromosome numbers in 51 oocyte germinal vesicles (GVs) from four triploid (diploid female $\times$ tetraploid male) females (top) and those in 25 GVs from five triploid (tetraploid female $\times$ diploid male) females (bottom) in loach Misgurnus anguillicaudatus


Triploid spermatocytes were screened by silver staining, differential $\mathrm{CMA}_{3}$ staining or FISH profiles to detect the presence of three homologous chromosomes bearing NORs in meiotic spread, because diploid and tetraploid loaches have two and four homologous chromosomes with NORs, respectively ( $\mathrm{Li}$ et al. 2010, 2011). Three Ag-NOR, $\mathrm{CMA}_{3}$ positeive sites or FISH signals were detected in 34 out of
91 meiotic cells examined. Other 57 cells demonstrated one or two NORs or rDNA loci. Among 34 putative triploid spermatocytes, 19 cells demonstrated two signals in one bivalent with tail-to-tail association of long arm of two homologous chromosomes and one signal in one univalent were dominant (Table 1; Fig. 4ab). Rarely, three cells showed one Ag-NOR staining or one $\mathrm{CMA}_{3}$-positive site at 



Fig. 3 Number of univalents (I), bivalents (II) and trivalents (III), and chromosome numbers in 163 spermatocytes from five triploid (diploid female $\times$ tetraploid male) males (top) and those in 264

just intermediate position of one bivalent and one signal in one univalent (Table 1; Fig. 4c). The signal in bivalent presumably included two NORs or rDNA loci formed by head-to-head association of short arm of two homologous chromosomes (Fig. 4c). On the contrary, in other 12 meiotic cells, three independent chromosomes (presumably univalents) gave Ag-NOR, $\mathrm{CMA}_{3}$-positive sites or FISH signals of rDNA loci (Table 1; Fig. 4d). In these cells, two homologous chromosomes could not be paired, indicating the failure of synapsis in these triploid spermatocytes.

\section{Discussion}

Both in triploid GVs and spermatocytes $(3 n=75)$, the most frequent meiotic configuration comprised equal number of 25 bivalents and 25 univalents $(25 \mathrm{II}+25 \mathrm{I})$, which was explained by the pairing between two spermatocytes from five triploid (tetraploid female $\times$ diploid male) males (bottom) in loach Misgurnus anguillicaudatus

homologous chromosomes from 50 chromosomes to form $25 \mathrm{II}$ and the failure of synapsis of other 25 chromosomes to form 25I. Differential staining and FISH analyses also supported that two homologous chromosomes paired to one bivalents and additional one chromosome became univalent in typical triploid spermatocytes. From such GVs and spermatocytes, gametes with the theoretical mode at $1.5 \mathrm{n}$ (chromosome number 37 or 38) can be generated by equal segregation of 25 bivalents and random segregation of 25 univalents. Thus, aneuploid progeny with the theoretical chromosome mode at $2 n=62$ or 63 will be produced when the triploid fish with aneuploid gametes is backcrossed to wild-type diploid $(2 \mathrm{n}=50)$. The same configuration with $25 \mathrm{II}+25 \mathrm{I}$ was previously reported in triploid loaches produced by artificial inhibition of the second polar body after fertilization of wild-type diploids (Zhang and Arai 1999) and those arisen from the cross between gynogenetic diploid female induced from diploid eggs of
Table 1 Meiotic configurations of homologous chromosomes bearing nucleolar organizing regions (NORs) identified by silver staiing, CMA3-positive site and FISH probed by rDNA sequence in spermatocytes of triploid males

\begin{tabular}{lllrr}
\hline & 2 in 1II (tail-to-tail) & $\begin{array}{l}2 \text { in 1II (head-to-head) } \\
1 \text { in 1I }\end{array}$ & 3 in 3I & Sum \\
\hline Ag-NOR & in 1I & 2 & 5 & 13 \\
CMA3-positive & 6 & 1 & 3 & 16 \\
FISH & 12 & 0 & 4 & 5 \\
Sum & 1 & 3 & 12 & 34 \\
\hline
\end{tabular}



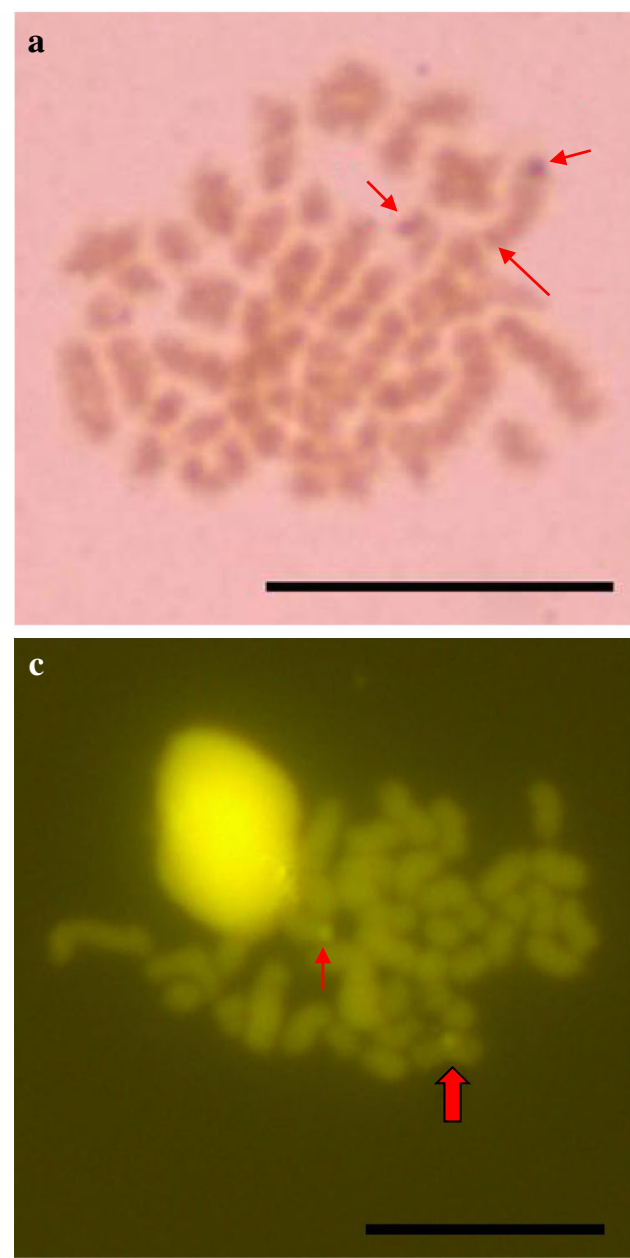

Fig. 4 Triploid spermatocytes showing one Ag-NOR site in one univalent and two $\mathrm{Ag}-\mathrm{NOR}$ sites in one bivalent with tail-to-tail association of homologous chromosomes (a), one $\mathrm{CMA}_{3}$-positive site in one univalent and two $\mathrm{CMA}_{3}$-positive sites in one bivalent with tail-to-tail association of homologous chromosomes (b), one $\mathrm{CMA}_{3}$ positive site in one univalent and one signal fused by two $\mathrm{CMA}_{3}-$ positive sites in one bivalent with head-to-head association of

spontaneously occurred tetraploid female and natural tetraploid male (Zhang et al. 2002). However, homologous chromosomes were not always synapsed in the course of meiosis and each of three homologous chromosomes behaved as univalent. These results suggest that misspairing might occur between homologous chromosomes in triploid meiotic cells, which results in the formation of unusual gametes by the involvement of many univalent in meiotic divisions.

In this study, presence of one, two, three or several trivalents (III) was observed in significant number of triploid meiotic cells. Trivalent chromosomes were detected by unusually long-rod like shape and triangular or ring-like shape probably due to the synapses among three homologous chromosomes. Thus, the appearance of trivalents may


homologous chromosomes (c), and one FISH signal probed by $5.8 \mathrm{~S}+28 \mathrm{~S}$ rDNA sequences in each of three univalents (d). NORs or rDNA loci shown by signals are indicated by arrows. NOR shown in the site just intermediate of one bivalent formed by head-to-head association of two homologous chromosomes is indicated by bold arrow. Scales denote $10 \mu \mathrm{m}$

relate to the presence of three sets of homologous chromosomes in triploid animals. No such trivalents have been seen in the meiosis of wild-type diploid loaches (Zhang et al. 1998; Yoshikawa et al. 2009; Li et al. 2011). However, all the triploids did not exhibit the configuration including trivalents: artificially induced triploids and previous triploids from diploid $\times$ tetraploid hybridization, they did not exhibit trivalents in the process of meiosis (Zhang and Arai 1999; Zhang et al. 2002).

As multivalent configuration, a few or several quadrivalents (IV) were detected together with many bivalents in meiotic cells of natural tetraploid loaches with four sets of chromosomes, but meiotic cells only with 25IV (100 chromosomes) have never appeared in tetraploid loaches ( $\mathrm{Li}$ et al. 2011). These previous results suggest that the 
formation of bivalents should take precedence of all other configurations like quadrivalents and trivalents even in polyploid germ cells. Relatively low frequencies of quadrivalents and predominance of bivalents were reported in meiosis of salmonid species which were considered as evolutionary tetraploidy (Nygren et al. 1972; Davisson et al. 1973; Lee and Wright 1981).

Presence of trivalents presumably makes the results of chromosome segregation more complicated, because each of two homologous chromosomes will be distributed to a different gamete in the case of bivalent, but distribution of homologous chromosome is presumably irregular in a trivalent: two chromosomes to one gamete and one chromosome to another or three chromosomes to one gamete and no chromosome to another.

In addition, many near triploid cells with extra and insufficient chromosome numbers were detected together with unusual polyploid (tetraploid to hexaploid range) cells. The mechanism responsible for the occurrence of aneuploid cells is unknown at present. Here, many polyploid cells were detected in spermatocytes of natural tetraploids. Such polyploid cells were considered to have resulted from spontaneous endomitosis (endoreduplication) (Li et al. 2011). Similar polyploid cells were also noticed in spermatocytes of salmonids (Nygren et al. 1972). Such unusual polyploids presumably due to endomitosis have been observed in the unreduced egg production process of triploid (Zhang et al. 1998) and clonal diploid (Itono et al. 2006), and in the unreduced sperm formation of sex-reversed clonal diploid (Yoshikawa et al. 2009) in the loach. Other possible mechanism responsible for the presence of polyploid spermatocytes may be the meiotic arrest after chromosome replication, followed by formation of spermatozoa or speramatozoon-like cells with unusual proceeding of spermiogenesis. Such kind of unusual spermatozoa or spermatozoon-like cells with duplicated DNA contents were reported in medaka hybrid male (Shimizu et al. 1997) and hyper-triploid loach (Zhao et al. 2014).

It was reported that artificially induced triploid loach males produced aneuploid spermatozoa presumably due to the meiotic configuration with $25 \mathrm{II}+25 \mathrm{I}$, but induced triploid females could not form fertile eggs (Zhang and Arai 1999). However, it was reported that induced triploid koi (ornamental carp) females spawned fertile eggs and then produced viable aneuploid (mean value 2.6n, range $2.3 n$ to $2.9 n$ ) progeny after crossing with diploid males (Gomelsky et al. 2015). Unexpected well-developed ovaries were also reported in common carp (Wu 1990; Cherfas et al. 1994). These previous studies suggest possible gonadal development and subsequent production of fertile gametes (eggs and sperm) even in triploid females from crosses between diploid and tetraploid Chinese loach, because meiotic configuration of $25 \mathrm{II}+25 \mathrm{I}$ giving rise to the theoretical mode of $1.5 \mathrm{n}$ gametes, which was reported in fertile induced triploid males (Zhang and Arai 1999), was predominant in both oocytes and spermatocytes in the present triploid loaches. Thus, further experimental studies are required to examine reproductive capacity of gametes generated by these triploid loach.

Acknowledgments This study was supported in part by KAKENHI Grant-in-Aid for Scientific Research (B) (No. 21380114) from the Japan Society of the Promotion of Science (JSPS) to K.A. and Grantsin-Aid from the Natural Science Foundation of China (No. 31272650) and the Natural Science Foundation of Liaoning Province (No. 201102019) to Y.J.L.

\section{References}

Arai K, Fujimoto T (2013) Genomic constitution and atypical reproduction in polyploidy and unisexual lineages of the Misgurnus loach, a teleost fish. Cytogenet Genome Res 140:226-240

Arai K, Mukaino M (1997) Clonal nature of gynogenetically induced progeny of triploid (diploid $\times$ tetraploid) loach Misgurnus anguillicaudatus (Pisces: Cobitididae). J Exp Zool 278:412-420

Arai K, Mukaino M (1998) Electrophoretic analysis of the diploid progenies from triploid $\times$ diploid crosses in the loach Misgurnus anguillicaudatus (Pisces: Cobitidae). J Exp Zool 280:368-374

Benfey TJ (1999) The physiology and behavior of triploid fishes. Rev Fish Sci 7(1):39-67

Benfey TJ (2011) Physiology of triploid fish. In: Farrell AP (ed) Encyclopedia of Fish Physiology: From Genome to Environment, vol 3. Academic Press, San Diego, pp 2009-2015

Cherfas NB, Gomelsky B, Ben-Dom N, Peretz Y, Hulata G (1994) Assessment of triploid common carp (Cyprinus carpio L.) for culture. Aquaculture 127:11-18

Davisson MT, Wright JE, Aterton LM (1973) Cytogenetic analysis of pseudolinkage of LDH loci in the teleost genus Salvelinus. Genetics 73:645-658

Gomelsky B, Schneider KJ, Anil A, Delomas TA (2015) Gonad development in triploid ornamental koi carp and results of crossing triploid females with diploid males. $\mathrm{N}$ Am J Aquac 77:96-101

Itono M, Morishima K, Fujimoto T, Bando E, Yamaha E, Arai K (2006) Premeiotic endomitosis produces diploid eggs in the natural clone loach, Misgurnus anguillicaudatus (Teleostei: Cobitidae). J Exp Zool A Comp Exp Biol 305A:513-523

Itono M, Okabayashi N, Morishima K, Fujimoto T, Yoshikawa H, Yamaha E, Arai K (2007) Cytological mechanisms of gynogenesis and sperm incorporation in unreduced diploid eggs of the clonal loach, Misgurnus anguillicaudatus (Teleostei: Cobitidae). J Exp Zool A Ecol Genet Phsiol 307A:35-50

Lee GM, Wright JE (1981) Mitotic and meiotic analyses of brook trout Salvelinus fontinalis. J Hered 72:321-327

Li YJ, Yin J, Wang JB, Yuan X, Wei J, Sun XW, Arai K (2008) A Study on the distribution of polyploid loaches in China. Nippon Suisan Gakkaishi 74:177-182

Li YJ, Tian Y, Zhang MZ, Tian PP, Yu Z, Abe S, Arai K (2010) Chromosome banding and FISH with an rDNA probe in the diploid and tetraploid Loach. Misgurnus anguillicaudatus. Ichthyol Res 57(4):358-366

Li YJ, Yu Z, Zhang MZ, Qian C, Abe S, Arai K (2011) The origin of natural tetraploid loach Misgurnus anguillicaudatus (Teleostei:Cobitidae) inferred from meiotic chromosome configurations. Genetica 139:805-811 
Li YJ, Zhang MZ, Qin C, Gao M, Arai K (2012) Fertility and ploidy of gametes of diploid, triploid and tetraploid loaches, Misgurnus anguillicaudatus, in China. J Appl Ichthyol 28:900-905

Li YJ, Yu Z, Zhang MZ, Qian C, Abe S, Arai K (2013) Induction and viable gynogenetic progeny using eggs and $\mathrm{UV}$-irradiated sperm from the Chinese tetraploid loach, Misgurnus anguillicaudatus. Aquacult Int 21:759-768

Matsubara K, Arai K, Suzuki R (1995) Survival potential and chromosomes of progeny of triploid and pentaploid females in the loach Misgurnus anguillicaudatus. Aquaculture 131:37-48

Morishima K, Horie S, Yamaha E, Arai K (2002) A cryptic clonal line of the loach Misgurnus anguillicaudatus (Teleostei: Cobitidae) evidenced by induced gynogenesis, interspecific hybridization, microsatellite genotyping and multilocus DNA fingerprinting. Zool Sci 19:565-575

Morishima K, Nakamura-Shiokawa Y, Bando E, Li YJ, Boron A, Khan MMR, Arai K (2008a) Cryptic clonal lineages and genetic diversity in the loach Misgurnus anguillicaudatus (Teleostei: Cobitidae) inferred from nuclear and mitochondrial DNA analyses. Genetica 132:59-69

Morishima K, Yoshikawa H, Arai K (2008b) Meiotic hybridogenesis in triploid Misgurnus loach derived from a clonal lineage. Heredity 100:581-586

Nygren A, Nilsson B, Jahnke M (1972) Cytological studies in Atlantic salmon from Canada, in hybrids between Atlantic salmon from Canada and Sweden and in hybrids between Atlantic salmon and sea trout. Hereditas 70:295-306

Piferrer F, Beaumont A, Falguiere J-C, Flajshans M, Haffray P, Colombo L (2009) Polyploid fish and shellfish: production, biology and applications to aquaculture for performance improvement and genetic containment. Aquaculture 293:125-156
Shimizu Y, Shibata N, Yamashita M (1997) Spermiogenesis without proceeding meiosis in the hybrid medaka between Oryzias latipes and O. curvinotus. J Exp Zool 279:102-112

Suzuki R, Nakanishi T, Oshiro T (1985) Survival, growth and sterility of induced triploids in the cyprinid loach Misgurnus anguillicaudatus. Bull Jpn Soc Sci Fish 51:889-894

Wu C (1990) Retrospects and prospects of fish genetics and breeding research in China. Aquaculture 86:111-125

Yoshikawa H, Morishima K, Fujimoto T, Saito T, Kobayashi T, Yamaha E, Arai K (2009) Chromosome doubling in early spermatogonia produces diploid spermatozoa in a natural clonal fish. Biol Reprod 80:973-979

Zhang Q, Arai K (1996) Flow cytometry for DNA contents of somatic cells and spermatozoa in the progeny of natural tetraploid loach. Fish Sci 62:870-877

Zhang Q, Arai K (1999) Aberrant meioses and viable aneuploidy progeny of induced triploid (Misgurnus anguillicaudatus) when crossed to natural tetraploids. Aquaculture 175:63-76

Zhang Q, Arai K, Yamashita M (1998) Cytogenetic mechanisms for triploid and haploid egg formation in the triploid loach Misgurnus anguillicaudatus. J Exp Zool 281:608-619

Zhang Q, Hanada K, Arai K (2002) Aberrant meioses in diploid and triploid progeny of gynogenetic diploids produced from eggs of natural tetraploid loach. Folia Zool 51:165-176

Zhao Y, Saito T, Pšenička M, Fujimoto T, Arai K (2014) Comparison of spermatozoa parameters, fine structures, and energy-related factors among tetraploid, hyper-tetraploid, and hyper-triploid loaches (Misgurnus anguillicaudatus). J Exp Zool A Ecol Genet Physiol 321A:198-206 